\title{
Dinâmica no uso e cobertura da terra na região do MATOPIBA entre 2000 e 2016
}

\author{
Eraldo Aparecido Trondoli MATRICARDI*, Thais Jacob MENDES ${ }^{1}$, Eder Miguel PEREIRA ${ }^{1}$, \\ Pedro Guilherme de Andrade VASCONCELOS ${ }^{1}$, Humberto ÂNGELO ${ }^{1}$, Olívia Bueno da COSTA ${ }^{1}$
}

\author{
${ }^{1}$ Departamento de Engenharia Florestal, Universidade de Brasília, Brasília, Distrito Federal Brasil. \\ *E-mail: ematricardi@gmail.com
}

Recebido em outubro/2018; Aceito em fevereiro/2019.

\begin{abstract}
RESUMO: O processo de ocupação das terras do Cerrado brasileiro tem impactado severamente sua paisagem natural nas últimas décadas. No presente estudo estimou-se as mudanças no uso e cobertura da terra ocorridas entre 2000 e 2016 na região do MATOPIBA usando dados e técnicas de sensoriamento remoto. Estimou-se também as áreas de agricultura irrigada e as alterações no uso e cobertura da terra ocorridas dentro de áreas protegidas na região de estudo. Nove classes de uso e cobertura de terra (corpos de água, cerrado denso, cerrado aberto, floresta ciliar, agricultura, campos irrigados, sombra, campos queimados e solo exposto) foram detectadas usando imagens de satélite e a técnica de classificação da máxima verossimilhança. Com base nos resultados desta pesquisa, observou-se o aumento 40,9\% de campos agrícolas e solos expostos entre 2000 e 2016 na área de estudo. As áreas de agricultura irrigada aumentaram aproximadamente 154\% (102.000 hectares) no mesmo período. Embora os diferentes tipos de antropização de áreas naturais tenham sido observados em toda a área de estudo, o maior aumento ocorreu na porção sul, onde as terras mais aptas para agricultura mecanizada estão mais concentradas. A antropização também aumentou dentro das terras protegidas de uso sustentável, onde as atividades de conservação deveriam ser prioritariamente implementadas na região de estudo, tais como a criação de corredores ecológicos e políticas públicas, visando o uso sustentável de seus recursos naturais.
\end{abstract}

Palavras-chave: desmatamento; Cerrado; sensoriamento remoto.

\section{Land use and land cover dynamic in the MATOPIBA region between 2000 and 2016}

\begin{abstract}
The land occupation process in the Brazilian Cerrado has severely impacted its natural landscape in the last decades. In this study, we estimated the land use and land cover changes occurred in the MATOPIBA region between 2000 and 2016 by using remotely sensed data and techniques. We also estimated the irrigated fields and deforested lands occurred within protected areas in the study region. Nine land use and land cover classes (water bodies, dense cerrado, open cerrado, riparian forest, agriculture, irrigated fields, shade, burned fields, and exposed soil) were detected by using Landsat imagery and by applying the Maximum Likelihood remote sensing technique. Based on our study results, we observed an increase of $40,9 \%$ agricultural fields and exposed soils during between 2000 and 2016 in the study region. Irrigated fields increased approximately $154 \%$ $(102,000 \mathrm{ha})$ during the studied period. Although different types of anthropization were observed throughout the study area, the major increasing change was observed within the southern portion, where the most suitable agricultural lands are concentrated. The anthropization also increased within protected lands of sustainable use, where conservation activities should be prioritarily enforced in the study region, such as the creation of ecological corridors and public policies aiming sustainable use of its natural resources.

Keywords: deforestation; Cerrado; remote Sensing.
\end{abstract}

\section{INTRODUÇÃO}

A região do MATOPIBA (Maranhão, Tocantins, Piauí e Bahia) é caracterizada pela expansão das atividades agrícolas de alta produtividade. Atualmente é uma das regiões mais promissoras do agronegócio no Brasil, apresentando safras crescentes de grãos, algodão e outros produtos oriundos da agricultura (MAGALHÃES; MIRANDA, 2014).

Paralelo ao destaque econômico e produtivo no cenário nacional tem-se a preocupação ambiental com a região do MATOPIBA, uma vez que $90 \%$ dela localiza-se no bioma cerrado e grande parte do desmatamento deste bioma está relacionado às atividades de expansão da produção de commodities agrícolas. Os processos de conversão de uso da terra no Cerrado contribuíram para reduzir mais da metade de sua vegetação natural (MIRANDA et al., 2014; BEUCHLE et al., 2015). Estima-se que o desmatamento no bioma Cerrado é maior do que na Amazônia (SPERA et al., 2016).

O Cerrado apresenta uma grande riqueza de espécies, diversidade de habitats e diferentes fitofisionomias, tornandoo a savana mais rica do mundo (RATTER et al., 1997; MMA; IBAMA, 2011). Além de possuir ampla diversidade biológica, o Cerrado presta serviços ambientais essenciais na regulação do ciclo do carbono e do ciclo hidrológico, uma vez que as principais cabeceiras de bacias hidrográficas brasileiras, como as bacias do Tocantins e do São Francisco, estão situadas nesse bioma (LAHSEN et al., 2016).

Apesar da sua dimensão e relevância, o Cerrado é um dos ecossistemas mais ameaçados do planeta devido aos impactos antrópicos sobre sua área e por isso recebeu o título de hotspot mundial tornando este bioma uma área prioritária para 
conservação na busca de garantir sua existência e continuidade (MYERS et al., 2000; FERREIRA; FREIRE, 2009).

Aproximadamente $46 \%$ da vegetação nativa desse bioma havia sido convertido em algum tipo de uso antrópico até 2017 (INPE, 2018). Além disso, diferente de outros biomas, a legislação ambiental é mais branda para este bioma (reserva legal deve apresentar no mínimo $20 \%$ da área da propriedade), permitindo exploração de extensas áreas (SPERA et al., 2016). Somado a isso, as áreas protegidas ocupam apenas $8,3 \%$ desse bioma, sendo que somente $6,5 \%$ dessas áreas é coberta por vegetação nativa, o restante já se encontra desmatado (FRANÇOSO et al., 2015).

O processo de ocupação dos solos tem provocado, nas últimas décadas, transformações drásticas em sua paisagem natural. No período de 2003 a 2013 a área de cultivo aumentou 2,3 milhões de hectares, ocupando, em sua maioria, áreas de Cerrado (SPERA et al., 2016). Essa transformação da vegetação nativa em desmatamento tem alto custo ambiental, causando fragmentação, poluição da água, degradação, alterações no regime do fogo, modificação do regime climático (KLINK; MACHADO, 2005) e perdas de solo por erosão (OLIVEIRA et al., 2015).

A região do MATOPIBA está sob risco de queda substancial da precipitação (entre 10 e $20 \%$ ) nos próximos anos, uma realidade que contrasta com o aumento da demanda de recursos hídricos, tendo em vista o crescimento da agricultura e das áreas irrigadas (IPCC, 2014). Estudos revelaram aumentos graduais na temperatura anual, frequência de dias quentes e diminuição da evapotranspiração na região do MATOPIBA, contundentes com as ações antropogênicas que ocorrem na região (NÓBREGA et al., 2017; SALVADOR; BRITO, 2018).

Neste sentido, o monitoramento sistemático e operacional destas áreas faz-se necessário a fim de garantir a preservação dos ecossistemas e, consequentemente, a manutenção dos ciclos biogeoquímicos e dos sistemas climáticos fundamentais para região. O uso de dados temporais combinados as técnicas de interpretação e processamento de imagens tem substituído os métodos tradicionais para o monitoramento da vegetação natural, pois permite identificar os processos de conversão destas áreas em agricultura, estimar taxas de desmatamento e a dinâmica do uso e cobertura da terra ao longo do tempo (SILVA et al., 2010).

O presente estudo buscou estimar as mudanças do uso e cobertura do solo na região do MATOPIBA a partir de dados de sensores remotos e técnicas de geoprocessamento nos anos de 2000, 2007 e 2016. A partir deste mapeamento, estimou-se também as áreas antropizadas dentro das Unidades de conservação e as áreas de agricultura irrigada na área de estudo. Os resultados desta pesquisa poderão apoiar a definição de estratégias e monitoramento do Cerrado brasileiro.

\section{MATERIAL E MÉTODOS}

\section{1. Área de Estudo}

A área de estudo (Figura 1) compreende porções de quatro estados brasileiros, leste do Tocantins, sudoeste do Piauí, sul do Maranhão e oeste da Bahia, localizado entre os meridianos $47^{\circ} 30^{\prime}$ e $44^{\circ} 00^{\prime}$ de longitude oeste e os paralelos $6^{\circ} 25^{\prime}$ e $15^{\circ}$ $05^{\prime}$ de latitude Sul.

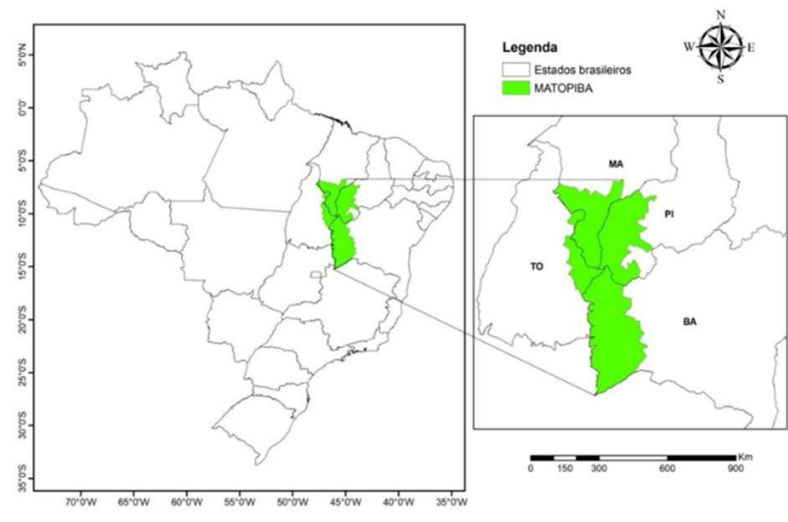

Figura 1. Localização da área de estudo.

Figura 1. Study area location.

A área de estudo está inserida no bioma Cerrado e possui como fitofisionomia dominante o cerrado arborizado que inclui os cerrados ralo, típico e denso (RIBEIRO; WALTER, 1998). Os solos do tipo latossolo são dominantes área de estudo no MATOPIBA, seguidos pelos neossolos e os plintossolos (MAGALHÃES; MIRANDA, 2014). O conjunto de planaltos com topos aplainados unidos à extensa rede hidrográfica e aos solos antigos, profundos e bem drenados, tornam a região propícia à implantação da agricultura moderna (SANTOS et al., 2011).

A região da área de estudo está inserida dentro de partes de três bacias hidrográficas: Bacia do Rio Tocantins, Bacia do Atlântico - Trecho Norte/Nordeste e Bacia do Rio São Francisco. Nestas bacias hidrográficas estão inseridos o Rio Tocantins, o Rio Araguaia, o Rio São Francisco e o Rio Parnaíba (MAGALHÃES; MIRANDA, 2014).

$\mathrm{Na}$ extensão do MATOPIBA predomina o clima tropical semiúmido, com temperaturas médias acima de $18^{\circ} \mathrm{C}$ em todos os meses do ano e precipitação média anual de $1.200 \mathrm{~mm}$ com períodos de seca bem definidos com duração de 4 a 5 meses (IBGE, 2012).

\subsection{Base de Dados}

As imagens utilizadas no estudo foram obtidas na base de dados do Serviço Geológico dos Estados Unidos (USGS). As imagens selecionadas foram as dos satélites Landsat 5 e 8, sensores TM (Thematic Mapper) e OLI (Operational Land Imager), para os anos 2000, 2007 e 2016 no período de julho a setembro (meses os quais as imagens apresentaram a menor quantidade de nuvens) (Tabela 1).

Tabela 1. Imagens dos satélites utilizadas para a classificação do uso e cobertura da terra.

Table 1. Satellite imagery used for land use/ land cover classification.

\begin{tabular}{cccc}
\hline Satélite/sensor & Ano & Path/row & Data \\
\hline & & $219 / 65-70$ & $12 / 08 / 2000$ \\
Landsat-5 / TM & \multirow{2}{*}{2000} & $220 / 65-69$ & $19 / 08 / 2000$ \\
& & $220 / 70$ & $03 / 08 / 2000$ \\
& & $221 / 65-68$ & $09 / 07 / 2000$ \\
& & $219 / 65-66$ & $17 / 08 / 2000$ \\
Landsat-5 / TM & \multirow{2}{*}{2007} & $220 / 65-70$ & $01 / 09 / 2007$ \\
& & $221 / 65-68$ & $07 / 08 / 2007$ \\
& & $222 / 65-66$ & $21 / 08 / 2007$ \\
Landsat-8 / OLI & \multirow{2}{*}{2016} & $220 / 65-70$ & $08 / 08 / 2016$ \\
& & $221 / 65-68$ & $14 / 07 / 2016$ \\
& & $222 / 65-66$ & $28 / 08 / 2016$ \\
& & &
\end{tabular}


Posteriormente, as imagens foram corrigidas radiometricamente e geometricamente para assegurar a fidelidade na análise espaço-temporal e a composição de mosaicos para a área de estudo. A correção radiométrica consistiu na transformação dos números digitais em valores físicos de reflectância no topo da atmosfera (TOA) (CHANDER et al., 2009). A correção geométrica foi realizada com polinômio de primeira ordem e reamostragem pelo vizinho mais próximo com tolerância de 0,5 pixel (DAI; KHORRAM, 1998).

\subsection{Classificação do uso e cobertura}

A classificação do uso e cobertura da terra foi feita a partir da inspeção visual e classificação supervisionada das cenas Landsat que recobrem a área de estudo, utilizando o software de geoprocessamento ERDAS IMAGINE®. Na etapa da inspeção visual, foram considerados os elementos de imagem como a cor para cada composição colorida RGB (Red, Green, Blue), a textura, a forma, o tamanho, a sombra, a altura e o padrão da drenagem e relevo. Cada tipo de uso e cobertura da terra foi identificado a partir da interpretação de todos estes elementos.

Para compor as imagens foram utilizadas as bandas 1 a $5 \mathrm{e}$ 7 do Landsat-5 e as bandas 2 a 7 do Landsat-8. A partir das imagens com as bandas compostas foi produzido um mosaico dos vários pontos (Row) para cada órbita (Path). Cada mosaico foi recortado para compor apenas os limites da área de estudo.

Nas visualizações preliminares, utilizou-se as imagens na composição colorida $5(\mathrm{R}) 4(\mathrm{G}) 3(\mathrm{~B})$ para as imagens do Landsat-5 e na $6(\mathrm{R}) 5(\mathrm{G}) 4(\mathrm{~B})$ para a imagem do Landsat- 8 com o intuito de realçar as áreas cobertas por vegetação fotossinteticamente ativa em tonalidade verde e rosa.

$\mathrm{Na}$ etapa da classificação supervisionada, as classes foram definidas as seguintes classes de interesse: Cerrado denso, Cerrado aberto, Solo exposto, Mata ciliar/galeria, Água (rios e corpos d'água), Queimadas e Agricultura. Além disso, foi necessária a criação de uma classe adicional (sombras) para diferenciar as sombras criadas nas proximidades de morros e serras de corpos de água. Esta classe foi criada e amostrada para aumentar a eficiência do classificador na diferenciação de áreas cobertas por água e áreas das sombras de morros e serras.

\subsubsection{Análise de Componentes Principais}

Após a definição preliminar das classes de interesse nas imagens Landsat, optou-se por transformar as imagens multiespectrais dos satélites utilizando a Análise dos Componentes Principais (ACP). Essa transformação das imagens feita pela ACP pode ser aplicada em métodos de classificação de imagens, pois possibilita a melhor discriminação das assinaturas espectrais das classes de interesse, especialmente em classes com redundância na reflectância (LOUGHLIN, 1991).

Assim, no presente estudo, foi gerada uma nova imagem com seis Componentes Principais a partir das imagens multiespectrais Landsat, compostas por seis bandas espectrais, utilizando o software ERDAS ${ }$ 2011. Na etapa seguinte, as imagens dos Componentes Principais foram utilizadas como entrada de dados da classificação supervisionada.

\subsubsection{Classificação supervisionada}

A classificação supervisionada teve como ponto de partida a definição de assinaturas espectrais extraídas das amostras de cada classe de interesse nas imagens dos Componentes
Principais, excluindo o sexto componente (CP 6), por se tratar do componente com maior quantidade de ruído entre todos os componentes.

A partir das classes previamente definidas, várias amostras foram coletadas sobre as imagens resultantes da Análise dos Componentes Principais. Na amostragem de cada uso e cobertura da terra para a classificação supervisionada, foram considerados 9 tipos de uso e cobertura de interesse para categorização: Água, Cerrado denso, Cerrado aberto, Mata ciliar, Agricultura, Irrigação, Sombra, Queimadas e Solo exposto. Para cada classe de interesse foram amostrados 40 a 70 polígonos com o intuito de obter assinaturas espectrais de classes melhor representadas espectralmente na área de estudo. As classes Cerrado denso, Cerrado aberto e Mata ciliar foram agrupadas na classe Cerrado para a estimativa de remanescentes de vegetação nativa na área e período de estudo,

As assinaturas espectrais de cada classe foram utilizadas como entrada do algoritmo de Máxima Verossimilhança (Maximum Likelihood) para então classificar os tipos de uso e cobertura em toda a área de estudo, gerando uma imagem classificada. Este método foi aplicado de forma individual para cada mosaico de imagens ACP derivados das imagens Landsat.

A classificação por mosaico buscou minimizar os efeitos sazonais na classificação, resultantes das diferentes datas de aquisição das imagens. Desta maneira, foram feitas 4 classificações, uma para cada órbita e uma para cada ano, com exceção do ano de 2000 que foi necessária mais uma classificação para a orbita/ponto 220/70, pois foi adquirida em uma data diferente.

O resultado dessa etapa foi a classificação do uso e cobertura da terra da área do MATOPIBA nos anos 2000, 2007 e 2016. Para eliminar ruídos na classificação (pixels compondo uma classe isolada no contexto de uma classe maior), foi aplicado o filtro "Majority", com janela 5 x 5 pixels. A estimativa da área total de cada classe de uso e cobertura da terra foi feita de forma indireta a partir da contagem de pixels de cada classe multiplicado pela área efetiva de cada pixel $\left(900 \mathrm{~m}^{2}\right)$.

\subsection{3. Áreas Irrigadas}

As áreas irrigadas foram delimitadas em formato vetorial, utilizando interpretação visual das imagens multiespectrais do satélite adquiridas utilizando escalas variáveis e composições coloridas RGB 5/4/3 (Landsat-5) e 6/4/5 (Landsat-8). Para a delimitação das áreas irrigadas foram considerados os pivôs centrais juntamente com as áreas de irrigação convencional (não-pivôs).

As áreas de pivôs centrais apresentam forma circular, enquanto as não-pivôs apresentam forma predominantemente retangular e em alguns casos foram identificadas pela comparação de suas tonalidades e o contraste com as demais áreas de agricultura e vegetação nativa presentes nas imagens.

\subsection{Checagem de campo}

Os trabalhos de campo foram feitos entre julho e agosto de 2016. De um modo geral, as checagens de campo buscaram a observação individual das diferentes coberturas (fitofisionomias) e usos da terra na região de estudo. Complementarmente, foram checados individualmente os pontos amostrais da classificação digital feita a partir de dados de sensoriamento remoto adquiridos em 2016. Alguns pontos 
amostrais localizados muito distantes das estradas de acesso e que estavam inseridos numa classe de uso ou cobertura homogênea e de grande dimensão territorial, foram observados dentro das respectivas classes nas proximidades do acesso mais próximo possível do ponto amostral para reduzir dispêndio de tempo e recursos.

\subsection{Avaliação da acurácia da classificação}

A avaliação da acurácia do mapeamento do uso e cobertura da terra foi feita a partir de uma Matriz de Confusão, comparando as imagens classificadas com 230 pontos aleatórios checados em campo (referência). O tipo de uso e cobertura da terra para cada ponto foi checado durante campanha de campo realizada em julho e agosto de 2016 em toda a área de estudo. Para efeito de avaliação da acurácia da classificação aplicada neste estudo e devido ao interesse na estimativa da vegetação típica do cerrado, sem discriminar os diferentes tipos, foram agrupadas as classes "Cerrado denso" e "Cerrado aberto" em uma nova classe "Cerrado".

$\mathrm{Na}$ matriz de confusão, o elemento da linha i coluna $\mathrm{k}$ (elemento da diagonal, $\mathrm{i}=\mathrm{k}$ ) contém o número de pixels identificados pelo usuário como corretamente classificados. Os demais elementos da linha $\mathrm{i}$ fornecem o número e distribuição dos pixels que foram classificados erroneamente. Portanto, a acurácia da classificação para classe i é dada pelo número de pixels da diagonal dividido pelo número total de pixels de referência para esta classe. A acurácia geral é uma média da acurácia das classes expressa em percentagem (CONGALTON; GREEN, 2008).

Os 230 pontos aleatórios utilizados para a validação da classificação foram comparados, individualmente, à classificação do uso e cobertura da terra de 2016. Esses dados foram utilizados para estimar a acurácia global e individual por classe de interesse do mapeamento.

A exatidão global para a matriz de confusão é calculada a partir da razão entre a soma dos valores da diagonal da matriz e a soma dos totais de suas linhas ou colunas (CONGALTON, 1991). O índice Kappa é baseado na diferença entre concordância observada (diagonal principal da matriz de confusão com a concordância entre a classificação e os dados de referência) e a chance de concordância entre os dados de referência e uma classificação aleatória (produto entre os totais marginais da matriz) (CONGALTON; GREEN, 2008).

\section{6. Áreas antropizadas em Unidades de Conservação}

Após a classificação das dezenove unidades de conservação presentes na área de estudo (Figura 2), a antropização (agricultura e solo exposto) foi quantificada para cada uma, sendo que nove dessas unidades representaram unidades de uso sustentável (atividades de coleta e uso dos recursos naturais são permitidas, desde que controladas e mantidas a sua sustentabilidade): APA do Rio Preto, APA de São Desidério, APA Serra da Tabatinga, APA do Jalapão, RPPN Catedral do Jalapão, APA das nascentes do Rio Vermelho, APA bacia do Rio de Janeiro e FLONA de Cristópolis; e dez representaram unidades de proteção integral (com uso apenas indireto e normas mais restritivas): PARNA Serra das Confusões, Parque Estadual do Jalapão, PARNA das nascentes do Rio Parnaíba, PARNA Grande Sertão Veredas, REVIS Veredas do oeste baiano, PARNA da Chapada das Mesas, MONA Canyons e corredeiras do Rio Sono, ESEC de Uruçuí-Uma, ESEC Serra Geral do Tocantins e ESEC do Rio Preto.

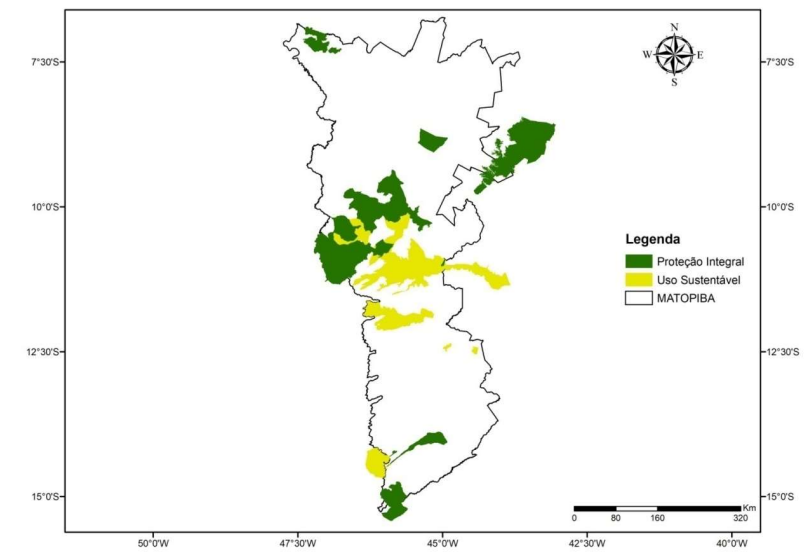

Figura 2. Localização das Unidades de Conservação na área de estudo.

Figure 2. Spatial location of the protected areas in the study area.

\section{RESULTADOS}

3.1. Avaliação da acurácia da classificação

De um total de 230 pontos amostrados aleatoriamente para a avaliação da acurácia, 184 foram classificados corretamente com base em checagem em campo, que resultou numa classificação digital do uso da terra utilizando imagens Landsat com exatidão global de $80 \%$ (Tabela 2). Similarmente, o coeficiente de Kappa foi de 0,76.

De um modo geral, os resultados da avaliação da acurácia do mapeamento indicam uma confiabilidade de moderada a alta para todas as classes. As classes "Agricultura" e "Cerrado" apresentaram bons índices de acurácia da classificação. Os resultados da avaliação da acurácia para irrigação foram mais exatos, pois esta classe foi delimitada a partir de classificação visual.

Tabela 2. Pontos amostrados usados para estimar a Matriz de Confusão Table 2. Sampled points used to estimate the Confusion Matrix.

\begin{tabular}{|c|c|c|c|c|c|c|c|c|c|}
\hline Classe & $\mathrm{AG}$ & $\mathrm{MC}$ & CR & SE & SO & IR & $\mathrm{QM}$ & AA & Total \\
\hline $\mathrm{AG}$ & 33 & & 4 & 3 & 0 & 0 & 0 & 0 & 40 \\
\hline $\mathrm{MC}$ & 2 & 22 & 9 & & 0 & 0 & 0 & 0 & 33 \\
\hline $\mathrm{CR}$ & 1 & 1 & 50 & 2 & 0 & 0 & 0 & 0 & 54 \\
\hline SE & 9 & & 1 & 25 & 0 & 0 & 0 & 0 & 35 \\
\hline SO & 0 & 5 & 1 & 0 & 11 & 0 & 0 & 3 & 20 \\
\hline IR & 0 & 0 & 0 & 1 & 0 & 23 & 0 & 0 & 24 \\
\hline QR & 0 & 2 & 1 & 0 & 0 & 0 & 16 & 1 & 20 \\
\hline AA & 0 & 0 & 0 & 0 & 0 & 0 & 0 & 4 & 4 \\
\hline Total & 45 & 30 & 66 & 31 & 11 & 23 & 16 & 8 & 230 \\
\hline
\end{tabular}

Códigos da tabela: $\mathrm{AG}=$ Agricultura; $\mathrm{MC}=$ Mata Ciliar; $\mathrm{CR}=\mathrm{Cerrado}$; $\mathrm{SE}=$ Solo exposto; $\mathrm{SO}=$ Sombra; $\mathrm{IR}=$ Irrigação; $\mathrm{QM}=$ Queimadas; $\mathrm{AA}=\mathrm{A} g u a$. 
A classe sombra apresentou uma alta superestimação. Entretanto, essa classe não possui grande relevância para o presente estudo, pois foi criada para facilitar a discriminação entre superfícies que pudessem ser confundidas pelo classificador.

3.2. Dinâmica do uso e cobertura da terra entre 2000, 2007 e 2016

Com base nos resultados da classificação supervisionada utilizada neste estudo, observou-se que houve expansão das áreas de agricultura sobre áreas originalmente cobertas por vegetação típica do Cerrado (Tabela 3; Figura 3). As áreas de agricultura (irrigada e não irrigadas) foram as principais alterações na paisagem mapeada, ocupando 4.217.466,2 ha, 4.763.678,9 ha e 5.943.749,0 ha em 2000, 2007 e 2016, respectivamente. Tais alterações no uso da terra implicam num crescimento de 40,9\% da área agrícola em 16 anos na área de estudo.

Extensas áreas antes cobertas por Cerrado até 2000 foram desmatadas e convertidas em áreas para cultivos agrícolas em 2016 (Figura 3). A intensidade de expansão agrícola observada na presente pesquisa também foi observada em outro estudo conduzido por Salmona et al. (2016) no oeste baiano. Estes autores estimaram que em oito anos (entre 2002 e 2010) mais de 1 milhão de hectares de vegetação nativa do Cerrado foram desmatadas naquela região de estudo.

Tabela 3. Uso e cobertura da terra em 2000, 2007 e 2016. Table 3. Land use and land cover in 2000, 2007, and 2016.

\begin{tabular}{lccc}
\hline \multirow{2}{*}{ Uso da terra } & \multicolumn{3}{c}{ Área (ha) } \\
\cline { 2 - 4 } & 2000 & 2007 & 2016 \\
\hline Queimadas & 454.929 & 1.041 .072 & 287.581 \\
Água & 39.779 & 41.143 & 40.806 \\
Sombra & 241.301 & 329.311 & 317.622 \\
Cerrado & 13.697 .933 & 12.346 .913 & 12.170 .777 \\
Solo exposto & 984.925 & 1.114 .215 & 1.044 .679 \\
Agricultura & 4.150 .865 & 4.652 .025 & 5.774 .868 \\
Irrigação & 66.601 & 111.654 & 168.882 \\
\hline Total & 19.636 .333 & 19.636 .333 & 19.636 .333 \\
\hline
\end{tabular}

Os resultados deste estudo indicam ainda que ocorreram sucessivas reduções na área de vegetação nativa do Cerrado (Cerrado denso, Cerrado aberto e Mata Ciliar) classificadas na área e período avaliado (Tabela 3).

As áreas de queimadas oscilaram abruptamente a cada ano de estudo, sendo que o fogo atingiu mais de 1 milhão de hectares em 2007. A ocorrência de áreas queimadas está relacionada sobretudo às práticas de uso e manejo da terra pela população da região, que incluem o uso do fogo para limpeza de terreno, pela facilidade e baixo custo (EMBRAPA, 2016).
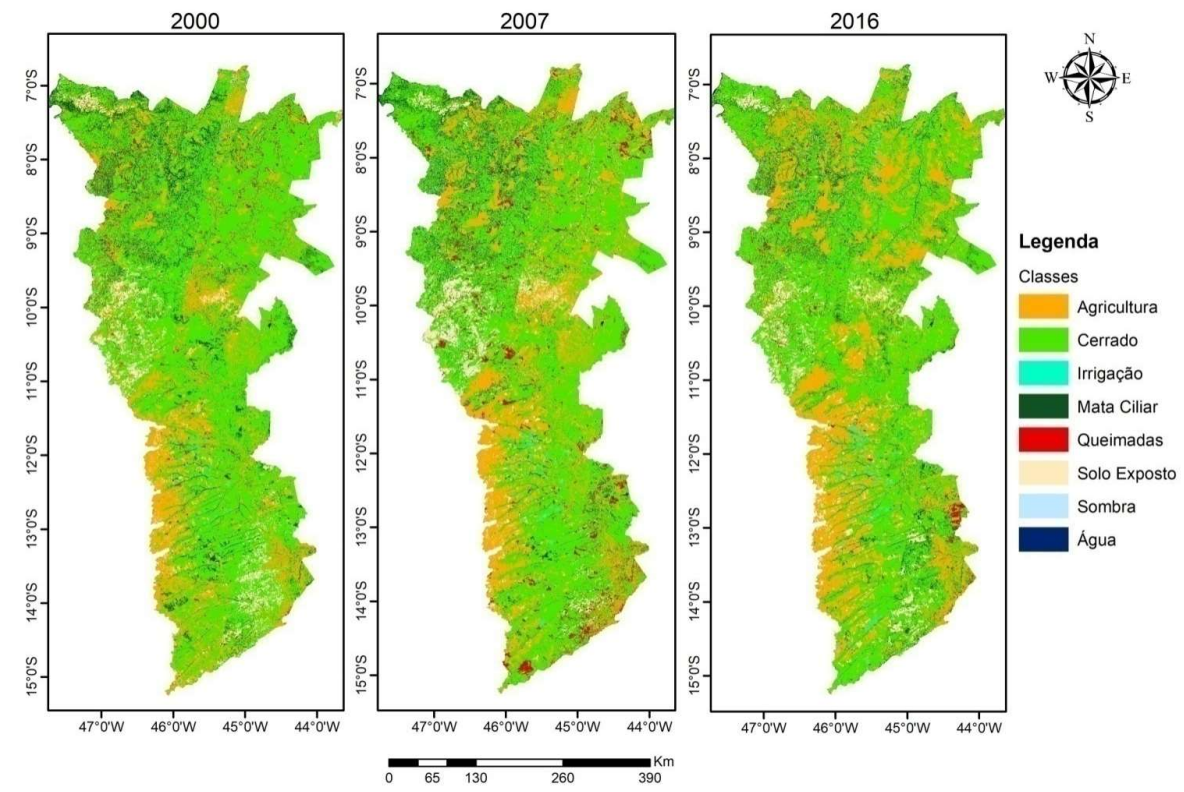

Figura 3. Mudanças no uso e cobertura da terra até 2000, 2007 e 2016.

Figure 3. Land use and land cover changes by 2000, 2007, and 2016.

As áreas de mata ciliar oscilaram de 1.774.359,6 ha para 1.242.936,9 ha entre 2000 e 2007 e, finalmente, para 1.505.105,7 ha em 2016. As áreas de solo exposto expandiram substancialmente no período e área de estudo. Os solos expostos ocupavam 984.925 ha e 1.044 .679 ha em 2000 e 2017, respectivamente. De acordo com observação feita em campo, estas áreas podem variar a cada ano devido aos diferentes estágios dos cultivos agrícolas praticados na região, que podem incluir uma fase de preparação do solo para o plantio, deixando o solo temporariamente exposto.

\section{3. Áreas Irrigadas}

As áreas irrigadas abrangiam 66.600,9 ha (2000), 111.654,3 ha (2007) e 168.881,5 ha (2016), sendo que os pivôs centrais ocupam quase que a totalidade dessas áreas.
Comparando com os anos do período analisado, as áreas irrigadas criadas na região resultaram em um aumento de aproximadamente 154\% (102.000 ha) em 16 anos (Tabela 3$)$. Com base nas observações de campo, estima-se que em torno de $90 \%$ das áreas irrigadas utilizam águas superficiais e 10\% águas subterrâneas. Dentro da área de estudo, os pivôs estão concentrados na região sul da área (Figura 4) que corresponde a porção do estado da Bahia com mais de $90 \%$ das áreas irrigadas identificadas.

As áreas de irrigação estão concentradas na zona do aquífero Urucuia (Figura 5) (ANA, 2018). Com base nas áreas irrigadas mapeadas neste estudo e nos limites das ottobacias nível 4 (Figura 6) elaborado por ANA (2018), observou-se atividades de irrigação agrícola nas seguintes sub-bacias: Rio Grande, Rio Preto, Rio Correntina, Rio Branco, Rio 
Carinhanha, Rio Guará, Rio do Meio, Rio Formoso, Rio Arrojado, Rio de Ondas e Rio das Fêmeas. As sub-bacias do Rio Branco, do Rio de Ondas e do Rio Formoso são, respectivamente, as bacias com a maior quantidade de áreas de agricultura irrigada.
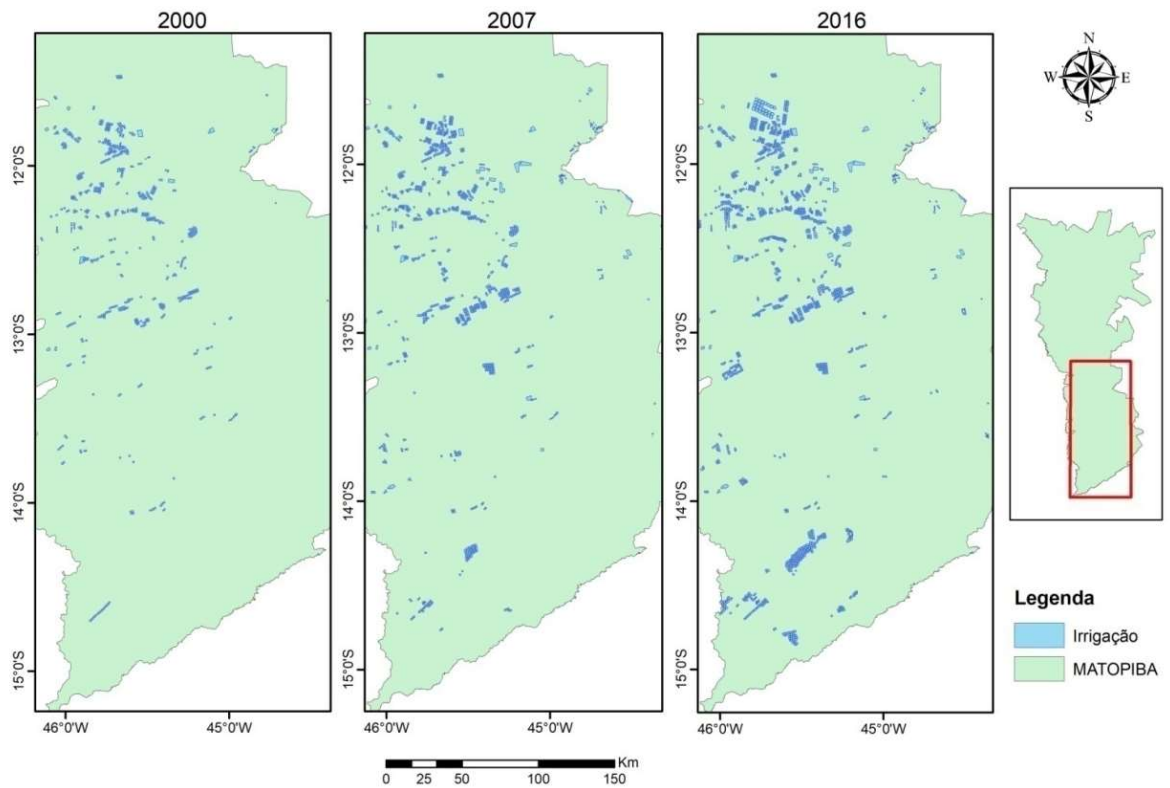

Figura 4. Áreas de irrigação detectadas na área de estudo em 2000, 2007 e 2016.

Figure 4. Irrigated crop lands detected in the study area in 2000, 2007, and 2016.

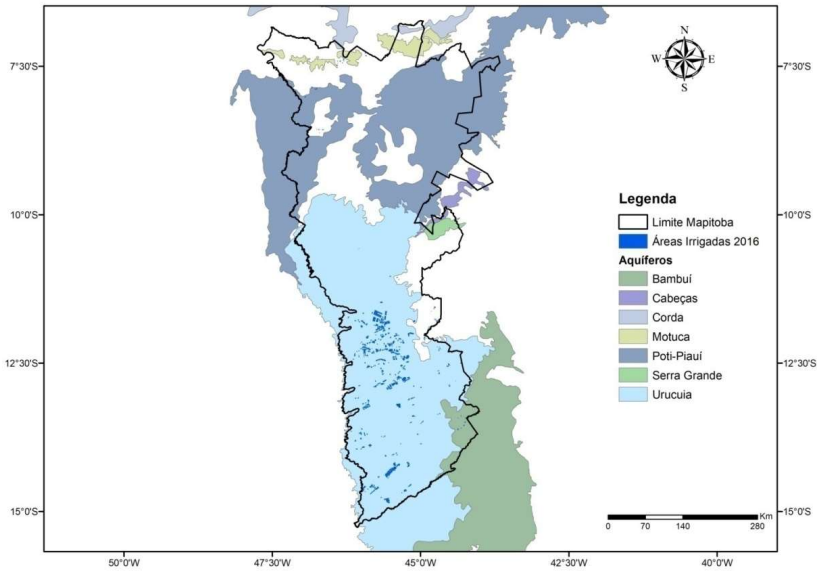

Figura 5. Principais aquíferos da área de estudo.

Figure 5. Main aquifers in the study area.

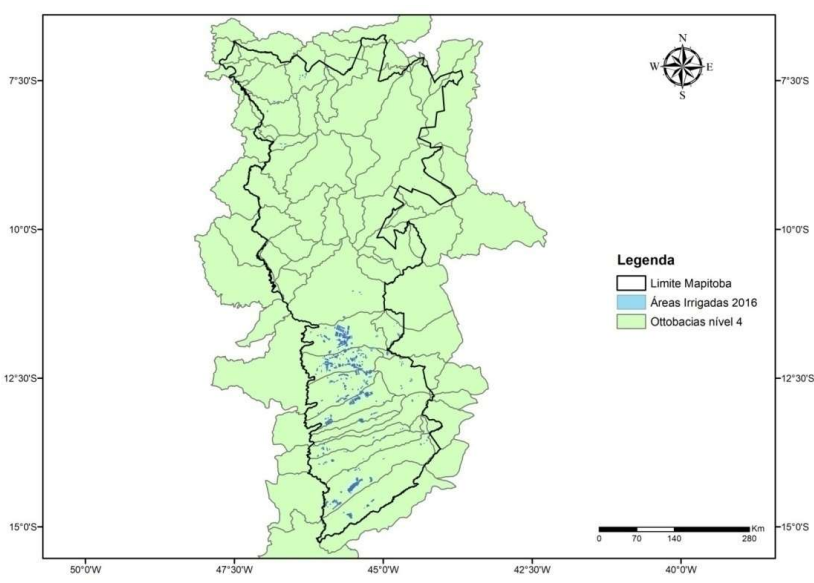

Figura 6. Ottobacias de nível 4 e áreas irrigadas.

Figure 6. Ottobacias level 4and the irrigated crop lands.

\subsection{Unidades de conservação}

Os resultados da classificação do uso e cobertura da terra para a área de estudo indicam que, em geral, houve redução da área total antropizada dentro das Unidades de Conservação de Proteção Integral (PI) e aumento da área total desmatada dentro das Unidades de Conservação de Uso Sustentável (US) (Tabela 4).

Tabela 4. Área antropizada dentro de unidade de conservação. Table 4. Anthropization area within protected areas.

\begin{tabular}{lccc}
\hline \multirow{2}{*}{$\begin{array}{c}\text { Unidade de } \\
\text { Conservação }\end{array}$} & \multicolumn{3}{c}{ Área antropizada (ha) } \\
\cline { 2 - 4 } & 2000 & 2007 & 2016 \\
\hline Proteção Integral & $291.985,9$ & $130.465,3$ & $117.912,2$ \\
Uso sustentável & $303.559,0$ & $365.219,6$ & $408.437,2$ \\
\hline Total & $595.544,9$ & $495.684,9$ & $526.349,4$ \\
\hline
\end{tabular}

Dentro das Unidades de Conservação de Proteção Integral, os desmatamentos mais expressivos foram observados dentro do Parque Nacional das Nascentes do rio Parnaíba, Estação Ecológica Serra Geral do Tocantins e, Parque Estadual do Jalapão. As maiores taxas de reduções (aproximadamente $91 \%, 82 \%$ e $68 \%$ ) de áreas antropizadas foram estimadas para o Parque Nacional Grande Sertão Veredas, Refúgio de Vida Silvestre das Veredas do oeste baiano e Parque Nacional da Chapadas das Mesas, respectivamente, entre 2000 e 2016 (Tabela 5). De forma oposta, a área antropizada aumentou dentro das Unidades de Conservação de Uso Sustentável. Os maiores aumentos (aproximadamente $330 \%$ e 170\%) de áreas antropizadas foram observados dentro das APA Rio Preto e Serra da Tabatinga, respectivamente.

As maiores áreas antropizadas até 2016 foram observadas dentro das APA do rio de Janeiro, do rio Preto e Serra da Tabatinga. 
Tabela 5. Dinâmica da antropização nas unidades de conservação

Table 5. Anthropization dynamic within the protected areas.

\begin{tabular}{|c|c|c|c|c|}
\hline \multirow[t]{2}{*}{ Unidade de Conservação } & \multicolumn{4}{|c|}{ Área antropizada (ha) } \\
\hline & Tipo & 2000 & 2007 & 2016 \\
\hline PARNA das nascentes do Rio Parnaíba & PI & 128.324 & 82.995 & 69.593 \\
\hline $\begin{array}{l}\text { Estação Ecológica Serra Geral do } \\
\text { Tocantins }\end{array}$ & PI & 44.263 & 9.689 & 17.014 \\
\hline Parque Estadual do Jalapão & PI & 19.033 & 1.251 & 8.140 \\
\hline Estação Ecológica de Uruçuí-Una & PI & 10.514 & 13.397 & 7.278 \\
\hline PARNA da Chapada das Mesas & PI & 21.919 & 3.334 & 6.983 \\
\hline PARNA Grande Sertão Veredas & PI & 44.355 & 11.348 & 4.134 \\
\hline REVIS Veredas do oeste baiano & PI & 20.388 & 6.893 & 3.673 \\
\hline PARNA Serra das Confusões & PI & 3.337 & 1.656 & 1.141 \\
\hline MONA canyons e corredeiras do Rio Sono & PI & 49,7 & 12 & 74 \\
\hline Estação Ecológica do Rio Preto & PI & 77 & 80 & 4,4 \\
\hline Total & PI & 292.260 & 130.655 & 118.034 \\
\hline APA bacia do Rio de Janeiro & US & 162.248 & 175.249 & 199.645 \\
\hline APA do Rio Preto & US & 42.748 & 175.576 & 182.498 \\
\hline APA Serra da Tabatinga & US & 9.907 & 16.566 & 26.863 \\
\hline Floresta Nacional de Cristópolis & US & 4.770 & 6.199 & 7602 \\
\hline APA do Jalapão & US & 12.301 & 1.226 & 5.132 \\
\hline APA de São Desidério & US & 2.441 & 3.804 & 2.741 \\
\hline RPPN Veredas do Pratudinho & US & 706 & 613 & 185 \\
\hline APA das nascentes do Rio Vermelho & US & 860,6 & 670,32 & 154 \\
\hline RPPN Catedral do Jalapão & US & 127,2 & 65,6 & 109 \\
\hline Total & US & 236.109 & 379.969 & 424.929 \\
\hline Total Geral & PI|US & 528.369 & 510.624 & 542.963 \\
\hline
\end{tabular}

\section{DISCUSSÃO}

Com base na metodologia de avaliação de acurácia proposta por Landis; Koch (1977), o mapeamento do uso e cobertura da terra conduzido no presente estudo pode ser considerado de boa acurácia, Isto indica que as estimativas das classes mapeadas nesta pesquisa podem ser utilizadas com boa exatidão para as inferências sobre as mudanças do uso e cobertura da terra ocorridas na região estudada.

A partir dos dados da classificação do uso e cobertura da terra, observou-se que houveram mudanças no uso da terra em toda a área de estudo. Entretanto, as principais alterações ocorreram na parte sul da área de estudo, onde existem extensas regiões de planaltos (chapadas) que que permitem o desenvolvimento de agricultura mais tecnificada na área de estudo (Figura 3).

Destacam-se também as alterações no uso da terra ocorridos no oeste baiano no período de estudo. Nessa região, devido às características topográficas e à adoção de projetos de irrigação visando suprir a escassez hídrica, ocorreu a expansão das grandes lavouras na região, sobretudo de soja, no período de 1984 a 2008 (PASSOS et al., 2010). A tendência de aumento do desmatamento do Cerrado é esperada nos próximos anos, a menos que hajam ações de controle, monitoramento e orientação técnica aos produtores rurais.

Extensas áreas atingidas por fogo foram observadas na área e período de estudo. O uso do fogo ainda é uma técnica de uso e manejo da terra na região e, portanto, a queima controlada pode fugir do controle se transformando em incêndios atingindo grandes áreas (EMBRAPA, 2016), como foi observado em 2007 no presente estudo, quando foram detectados mais de 1 milhão de hectares de áreas queimadas.

As áreas de mata ciliar oscilaram de $1.774 .359,6$ ha para 1.242.936,9 ha entre 2000 e 2007 e, finalmente, para 1.505.105,7 ha em 2016. As áreas de matas ciliares variaram ao longo do período estudado. Com base em observações de campo, é provável que o desmatamento e a variação sazonal tenham sido responsáveis pela variação das áreas mapeadas nesta classe de cobertura da terra.

A expansão das áreas de solo exposto está relacionada à expansão da agricultura na região de estudo. Embora algumas áreas de cerrado aberto e solo exposto possam ter sido confundidas pelo classificador, observou-se em campo que houve aumento substancial das áreas de solo exposto. Neste caso, a maioria das áreas com solos expostos estão associadas à expansão agrícola na região, especialmente durante o período de entressafras de sistemas de plantios convencionais, quando os agricultores preparam os solos para o cultivo da safra seguinte.

A ampliação das técnicas de plantios diretos na região do Cerrado brasileiro, mantendo a cobertura do solo com os restos vegetais da cultura anterior (ALMEIDA et al. 2018), poderá reduzir a quantidade dessas áreas de solos expostos na região estudada.

A concentração das áreas de irrigação sobre o aquífero Urucuia (Figura 5) indica a necessidade de controle mais efetivo da retirada de águas superficiais e subterrâneas neste aquífero. A preservação e recuperação de áreas ao redor de mananciais podem beneficiar a agricultura (responsável pelo uso de aproximadamente $70 \%$ de toda a água consumida no país), além de auxiliar outros serviços ambientais (BRANCALION et al., 2016). Entretanto, a supressão da vegetação nativa frente à expansão dos cultivos agrícolas, pode comprometer a manutenção de serviços ambientais, como a purificação da água, proteção do solo, regulação climática e falta de água (OLIVEIRA et al., 2015; BRANCALION et al., 2016).

Além disso, os impactos do aumento de áreas irrigadas incluem a compactação e erosão dos solos e o aumento da contaminação das águas com produtos agrícolas tóxicos (ALMEIDA; COSTA, 2014). O aumento de áreas irrigadas pode afetar as condições climáticas e diminuir as fontes de água potável, impactando o ciclo hidrológico (VRESE et al., 2016). 
As unidades de proteção integral possuem regras rígidas com relação a sua utilização (uso indireto), inferindo-se assim que a legislação não tem sido respeitada, pois foram observadas extensas áreas antropizadas dentro dessas Unidades de Conservação (Tabela 4). Apesar disso, houve redução do total antropizado nos últimos anos analisados, que pode ser o reflexo de atividades de fiscalização além de uma maior conscientização sobre a utilização sustentável do meio ambiente. Contudo, a partir dos resultados, é evidente que os esforços de conservação do Cerrado pelo Governo Brasileiro estão longes do ideal (FRANÇOSO et al., 2015).

As Unidades de Conservação de Uso Sustentável foram criadas com o propósito principal de compatibilizar a conservação da natureza com o uso sustentável dos recursos naturais. Por isso, estes tipos de Unidades possuem uma maior flexibilidade em relação à sua utilização comparados com as Unidades de Conservação de Proteção Integral. Por esta razão, as áreas antropizadas ocorreram de forma mais intensa dentro das Áreas de Proteção Ambiental. A intensidade de uso da terra deveria ser objeto de avaliação mais detalhada por parte dos órgãos gestores das respectivas áreas protegidas com o intuito de garantir a sustentabilidade ambiental dessas unidades.

O foco na proteção da floresta Amazônica fez com que no passado as políticas públicas negligenciassem a conservação do Cerrado. No entanto, existe atualmente grandes oportunidades para ações que envolvam vários setores da sociedade na busca da conservação e uso sustentável desse bioma. Devido ao grau de alteração e degradação já alcançados deveria ser dada prioridade à execução de ações que fortaleçam as áreas protegidas já existentes e que criem novas áreas de proteção (KLINK; MACHADO, 2005).

As áreas antropizadas observadas dentro das Estações Ecológicas de Uruçuí-Uma e Serra Geral do Tocantins foram expressivas. Tratam-se de Unidades de Conservação de proteção integral com uso da terra bastante restrito pela legislação e, portanto, não deveriam ter sido objeto de atividades antrópicas com as dimensões territoriais neste estudo.

Do mesmo modo, houve antropização expressiva nas APA do Rio Preto e do Rio de Janeiro. As maiores áreas antropizadas podem estar relacionadas à modalidade destas unidades de conservação que permite que sejam implementados diferentes usos da terra, conforme a legislação específica de sua criação.

\section{CONCLUSÕES}

A ampliação das áreas agrícolas e de solos expostos entre 2000 e 2016 na região do MATOPIBA ocorreu, em sua maioria, em detrimento de áreas ocupadas por vários tipos de vegetação típica do cerrado, incluindo a intensificação do antropismo dentro de Unidades de Conservação de uso sustentável. Ao mesmo tempo, houve recuo das atividades antrópicas no interior de Unidades de Conservação de proteção integral, consideradas cruciais para a preservação dos recursos naturais renováveis na região estudada.

$\mathrm{O}$ aumento substancial das áreas de plantios irrigados permite inferir que houve aumento da demanda e uso dos recursos hídricos superficiais e subterrâneos, considerados temas polêmicos na região de estudo e, portanto, devem ser devidamente considerados pelos órgãos gestores Recomendase a continuidade do monitoramento por satélite com o intuito de observar a dinâmica do uso e cobertura da terra na região do MATOPIBA e, partir disso, possibilitar a definição de políticas e estratégias mais adequadas para controlar e reduzir a perda de recursos naturais e biodiversidade nesta região.

\section{REFERÊNCIAS}

ALMEIDA, J. J. G. A.; COSTA, F. R. Análise dos impactos socioeconômicos e ambientais da agricultura irrigada no perímetro irrigado de Paus dos Ferros (RN). Revista Geografares, Vitória, n. 16, p. 22-44, 2014. DOI: https://dx.doi.org/10.7147/GEO16.4898

ALMEIDA, V.; ALVES JUNIOR, J., MESQUITA, M., EVANGELISTA, A.W.P., CASAROLI, D., BATTISTI, R. Comparação da viabilidade econômica da agricultura irrigada por pivô central em sistemas de plantios convencional e direto com soja, milho e tomate industrial. Global Science And Technology, v. 11, n, 02. p. 256-273, 2018.

ANA AGÊNCIA NACIONAL DE ÁGUAS. SISTEMA NACIONAL DE RECURSOS HÍDRICOS. Água Subterrânea. Brasília. Disponível em: http://www3.ana.gov.br. Acesso em: 20 dezembro de 2018

BEUCHLE, R.; GRECCHI, R. C.; SHIMABUKURO, Y.E.; SELIGER, R.; EVA, H. D.; SANO, E. E.; ACHARD, F. Land cover changes in the Brazilian Cerrado and Caatinga biomes from 1990 to 2010 based on a systemtic remote sensing samplig approach. Applied Geography, Oxford, v. $58, \quad$ p. $116-127, \quad 2015 . \quad$ DOI: https://dx.doi.org/10.1016/j.apgeog.2015.01.017

BRANCALION, P. H. S.; GARCIA, L. C.; LOYOLA, R.; RODRIGUES, R. R.; PILLAR, V. D.; LEWINSOHN, T. M. Análise crítica da Lei de Proteção da Vegetação Nativa (2012), que substituiu o antogo Código Florestal: atualizações em curso. Natureza e Conservação, v. 14, p. e1-e16, 2016.

DOI: https://dx.doi.org/10.1016/j.ncon.2016.03.004

CHANDER, G.; MARKHAM, B. L.; HELDER, D. L. Summary of current radiometric calibration coefficients for Landsat MSS, TM, ETM+, and EO-1 ALI sensors. Remote Sensing of Environment, New York, v. 113, n. 5, p. 893-903, 2009. DOI: https://dx.doi.org/10.1016/j.rse.2009.01.007

CONGALTON, R. G. A Review of assessing the accuracy of classifications of remotely sensed data. Remote Sensing of Environment, v. 37, n. 1, p. 35-46, 1991. DOI: https://dx.doi.org/10.1016/0034-4257(91)90048-B

CONGALTON, R. G.; GREEN, K. Assessing the accuracy of remotely sensed data. 2. ed. CRC Press, 2008. $183 \mathrm{p}$.

DAI, X.,KHORRAM, S. The effects of image misregistration on the accuracy of remotely sensed change detection. IEEE Transaction on Geoscience and Remote Sensing, v. 36, n. 5, p. 1566-1577, 1998

EMBRAPA_EMPRESA BRASILEIRA DE PESQUISA AGROPECUÁRIA. Aspectos motivacionais para o uso do fogo na agricultura no Distrito Federal e Entorno. Brasília: Embrapa, 2016. 109 p. (Boletim de Pesquisa e Desenvolvimento).

FERREIRA, M.; FREIRE, N. Community perceptions of four protected areas in the Northern portion of the Cerrado hotspot, Brazil. Environmental Conservation, Lausanne, v. 36 , n. 2, p. 129-138, 2009. DOI: https://dx.doi.org/10.1017/S0376892909990166 
FRANÇOSO, R. D.; BRANDÃO, R.; NOGUEIRA, C. C.; SALMONA, Y. B.; MACHADO, R. B.; COLI, G. R. Habitat loss and the effectiveness of protected areas in the Cerrado biodiversity hotspot. Natureza e Conservação, v. 13, n. 1, p. 35-40, 2015. DOI: https://dx.doi.org/10.1016/j.ncon.2015.04.001

IBGE_INSTITUTO BRASILEIRO DE GEOGRAFIA E ESTATÍSTICA. Mapa de Clima do Brasil. Disponível em: < http://www.vizualizador.inde.gov.br/ > . Acesso em: $09 / 11 / 2017$.

IPCC INTERGOVERNAMENTAL PANNEL ON CLIMATE CHANGE. Climate Change 2014. New York: PRESS, C.U.; 2014. 169p.

INPE_Instituto Nacional de Pesquisas Espaciais. Coordenação Geral de Observação da Terra. Projeto PRODES Cerrado - Incremento anual de área desmatada no Cerrado Brasileiro. Disponível em: http://www.obt.inpe.br/cerrado. Acesso em: 20 dezembro de 2018.

KLINK, C. A.; MACHADO, R. B. Conservation of the Brazilian Cerrado. Conservation Biology, Boston, v. 19, n. $3, \quad$ p. 707-713, 2005. DOI: https://dx.doi.org/10.1111/j.1523-1739.2005.00702.x

LAHSEN, M.; BUSTAMANTE, M. M. C.; DALLA-NORA, E. L. Undervaluing and overexploiting the Brazilian Cerrado at our peril. Environment: Science and Policy for Sustainable Development, Saint Louis, v. 58, n. 6, p. 4-15, 2016.2 DOI: https://dx.doi.org/10.1080/00139157.2016.1229537

LANDIS, J. R.; KOCH, G. G. The measurement of Observer Agreement for Categorical Data. Biometrics, Washington, v. $33, \quad$ n. $1, \quad$ p. $159-174,1977 . \quad$ DOI: https://dx.doi.org/10.2307/2529310

LOUGHLIN, W. P. Principal Component Analysis for Alteration Mapping. Photogrammetric Engineering and Remote Sensing, Vol. 57, n. 9, 1991, pp. 1163-1169. Disponível em: https://www.asprs.org/wpcontent/uploads/pers/1991journal/sep/1991_sep_11631169.pdf. Acesso em: 22 abril de 2019.

MAGALHÃES, L. A.; MIRANDA, E. E. Matopiba: quadro natural. Campinas, SP: Embrapa, 2014. 41p. (Nota Técnica 5).

MIRANDA, E. E.; MAGAlHÃES, L. A.; CARVALHO, C. A. Proposta de delimitação territorial do MATOPIBA Campinas, SP: EMBRAPA, 2014. 18 p.

MMA MINISTÉRIO DO MEIO AMBIENTE; IBAMA O INSTTITUTO BRASILEIRO DO MEIO AMBIENTE E DOS RECURSOS NATURAIS RENOVÁVEIS. Projeto Monitoramento do desmatamento nos biomas brasileiros por satélite. Monitoramento do bioma Cerrado 2009-2010. Brasília: MMA, 2011. 65 p.

MYERS, N.; MITTERMEIER, R. A.; DA FONSECA, G. A.; KENT, J. Biodiversity hotspots for conservation priorities. Nature, London, v. 403, p. 853-858, 2000. DOI: https://dx.doi.org/10.1038/35002501
NÓBREGA, R. L. B.; GUZHA, A. C.; TORRES, G. N.; KOVACS, K.; LAMPARTER, G.; AMORIM, R. S. S.; COUTO, E.; GEROLD, G. Effects of conversion of native cerrado vegetation to pasture on soil hydro-physical properties, evapotranspiration and steamflow on the Amazonian agricultural frontier. PlosOne, v. 12, n. 6, p. 122 , 2017.

DOI: https://dx.doi.org/10.1371/journal.pone.0179414

OLIVEIRA, P. T. S.; NEARING, M. A.; WENDLAND, E. Orders of magnitude increase in soil erosion associated with land use change from native to cultivated vegetation in a Brazilian savannah envoronment. Earth surface processes and landforms, Sussex, v. 40, p. 1524-1532, 2015. DOI: https://dx.doi.org/10.1002/esp.3738

PASSOS, A. L. O.; ROCHA, S. S.; HADLICH, G. M. Evolução do uso do solo e agronegócio na região oeste do estado da Bahia. Cadernos de Geociências, n. 7, p. 31-39, 2010.

RATTER, J. A.; RIBEIRO, J. F.; BRIDGEWATER, S. The Brazilian Cerrado Vegetation and threats to its biodiversity. Botanical Briefing, v. 80, p. 223-230, 1997. DOI: https://dx.doi.org/10.1006/anbo.1997.0469

RIBEIRO, J. F.; WALTER, B. M. T. Fitofisionomias do bioma Cerrado. In: SANO, S. M.; ALMEIDA, S.P. (Ed.). Cerrado: ambiente e flora. Brasília: Embrapa Cerrados, 1998. p. 89-166

SALMONA, Y. B.; PAIVA, A. O.; MATRICARDI, E. A. T. Estimativas futuras de desmatamento e emissões de $\mathrm{CO}_{2}$ equivalente no Oeste Baiano. Revista Brasileira de Cartografia, Rio de Janeiro, v. 68, n. 7, p. 1385-1395, 2016.

SALVADOR, M. A.; BRITO, J. I. B. Trend of annual temperature and frequency of extreme events in the MATOPIBA region of Brazil. Theoretical and Applied Climatology, Wien, v. 133, n. 1-2, p. 1-9, 2017. DOI: https://dx.doi.org/10.1007/s00704-017-2179-5

SANTOS, H. G. dos; CARVALHO JUNIOR, W. de; DART, R. de O.; AGLIO, M. L. D.; SOUSA, J. S. de; PARES, J. G.; FONTANA, A.; MARTINS, A. L. da S.; OLIVEIRA, A. P. de. O novo mapa de solos do Brasil. Rio de Janeiro, RJ: Embrapa Solos, 2011. 67 p. (Documentos 130).

SILVA, G. B. S.; FORMAGGIO, A. R.; SHIMABUKURO, Y. E.; ADAMI, M.; SANO, E. E. Discriminação da cobertura vegetal do Cerrado matogrossense por meio de imagens MODIS. Pesquisa Agropecuária Brasileira, Brasília, v. 45, n. 2, p. 186-194, 2010. DOI: http://dx.doi.org/10.1590/S0100-204X2010000200010

SPERA, S. A.; GALFORD, G. L.; COE, M. T.; MACEDO, M. N.; MUSTARD, J. F. Land-use change affects water recycling in Brazil's last agricultural frontier. Global Change Biology, Oxford, v. 22, p. 3405-3413, 2016. DOI: https://dx.doi.org/10.1111/gcb.13298

VRESE, P.; HAGEMANN, S.; CLAUSSEN, M. Asian irrigation, Africa rain: remote impacts of irrigation. Geophysical Research lettters, v. 43, n. 8, p. 3737-3745, 2016. DOI: https://dx.doi.org/10.1002/2016GL068146 